\title{
Mechanical Properties Measurement of Polymer Films by Bulge Test and Fringe Projection
}

\author{
C. S. Lin, ${ }^{1}$ T. L. Horng, ${ }^{2}$ J. H. Chen, ${ }^{3}$ K. H. Chen, ${ }^{4}$ J. J. Wu, ${ }^{5}$ C. Y. Chen, ${ }^{6}$ and S. H. Ma ${ }^{3}$ \\ ${ }^{1}$ Ph. D. Program of Mechanical and Aeronautical Engineering, Feng Chia University, No. 100 Wen Hwa Road, Taichung 40724, Taiwan \\ ${ }^{2}$ Department of Applied Mathematics, Feng Chia University, No. 100 Wen Hwa Road, Taichung 40724, Taiwan \\ ${ }^{3}$ Department of Photonics, Feng Chia University, No. 100 Wen Hwa Road, Taichung 40724, Taiwan \\ ${ }^{4}$ Department of Electrical Engineering, Feng Chia University, No. 100 Wen Hwa Road, Taichung 40724, Taiwan \\ ${ }^{5}$ Department of Environmental Engineering and Science, Feng Chia University, No. 100 Wen Hwa Road, Taichung 40724, Taiwan \\ ${ }^{6}$ Department of Material Science and Engineering, Feng Chia University, No. 100 Wen Hwa Road, Taichung 40724, Taiwan
}

Correspondence should be addressed to T. L. Horng; thorng123@gmail.com

Received 25 March 2014; Accepted 21 April 2014; Published 20 May 2014

Academic Editor: Chi-Wai Chow

Copyright (C) 2014 C. S. Lin et al. This is an open access article distributed under the Creative Commons Attribution License, which permits unrestricted use, distribution, and reproduction in any medium, provided the original work is properly cited.

\begin{abstract}
This paper aims at determining the mechanical properties such as Poisson ratio, biaxial modulus, and residual stress of polymer membranes using the bulge test and fringe projection. Under this idea, a bulge test apparatus has been developed for robust usage, under the room temperature, in industrial environments that are usually noisy and full of all kinds of vibrations. A setup of the bulge test, consisting of single chips and digital on-off valves, was used to achieve automatic precision control of pressure application to gas chamber with a circular polyimide (PI) test film fixed on the opening of it. A deformed image was then obtained via fringe projections with a series of optical apparatuses, and a 3D membrane deformation distribution was further achieved from this deformed image by some image processes that are chiefly through wavelet transformation and phase expansion. With the measurements of maximum membrane deformation versus applied pressure and given the Young's modulus of test membrane, we could further calculate the Poisson ration (and then biaxial modulus) and residual stress of the film. Also, the measurements were validated to be highly accurate by comparison with a finite element analysis.
\end{abstract}

\section{Introduction}

Nowadays, polyimide (PI) films have become a popular material because they are thin, light, flexible, and high-impact resistant $[1,2]$. Also, PI films have been wildly applied to replace glass material in liquid crystal displays in the trend of using soft display, flexible printed and integrated circuits, and memory devices. PI films are generally manufactured by a roll-to-roll process, which might affect their mechanical properties and cause unwanted residual stresses that would significantly degrade the quality of display. A variety of methods can be used to analyze the mechanical properties of thin films, such as optical fringe, nanoindentation test, tensile test, bulge test, microcantilever bending test, and acoustic resonance inspection [3-8]. Among methods mentioned above, the frequently used tensile test can only measure Young's modulus. Additional important mechanical properties, such as biaxial modulus and residual stresses, are mostly measured by bulge test via the relationship of pressure applied and film deformation. Therefore, accurate measurements of film deformation and precise control of pressure application are key technologies for bulge test.

Many methods, such as laser interferometry, atomic force microscopy, white light interferometry, mechanical profilometry, Michelson interferometry, coherent gradient detection, and electronic speckle interferometry, have been proposed to measure film deformation [9]. Since these methods all use interferometry, they are very sensitive to environmental noises and vibrations. Hence, the measurement range and resolution could thus be limited, and using these methods in generally noisy industrial environments could be difficult. By contrast, moiré fringes projection technology has 
the advantage of resistance to the environmental disturbances and is widely used for 3D surface measurements [10]. This motivates the current study to adopt fringe projection for the measurement of film deformation.

As mentioned above, precise pressure controlling is important for bulge test. Generally, pressure controlling system can be divided as hydraulic pressure system and pneumatic pressure system. The incompressibility of hydraulic system would make the pressurization slow but stable, which is easier for accurate control. However, if the entrained bubbles inside the hydraulic fluid are not thoroughly removed, it would cause air lock and therefore nonuniform pressurization of membrane [11]. As to the pressure regulation, it can be done either by servo or manual control. Using feedback control, servo system has the merits of accuracy and stability but is also pricy. On the contrast, manual control is less accurate but more economic.

Considering all factors mentioned above, here we propose an accurate, efficient, and economic bulge test equipment, basically consisting of an automatic pneumatic pressure regulating system, a fringe projection system, and an image capturing and processing system to measure the Poisson ration (and then biaxial modulus) and residual stresses of PI films. Young's modulus, biaxial modulus, and Poisson ratio are basic mechanical properties of thin films, which tell you the elastic modulus and hardness of a thin film. These properties further reveal the load-displacement curve of a thin film. As to the residual stresses, They are usually low in the manufacturing of thin films. However, if it is large, it will cause deformation and defect, which would further affect the reliability and stability of thin films in application. To elaborate, we used nitrogen as the working gas in our pneumatic system. It has the advantage of low density, requests smaller driving force, and responds quickly to deform the membrane. A single-chip microprocessor was deliberately used for the automatic pressure regulation in order to avoid dramatic changes of deformation of the test membrane.

In the optical fringe projection system, a specially installed telecentric lens, together with four-step phase shift method, was employed for the projection in order to retrieve accurate and real-time phase data of the deformed surface. The phase data was further captured, processed, and transformed into the distribution of film deformation by the image capturing and processing system.

Through our equipment, data of maximum deformation versus pressure was measured and curve-fitted into a cubic polynomial. From it, together with Young's modulus measured by microtensile test in advance, the Poisson ratio (and then biaxial modulus) and residual stresses can then be determined. Here, we also verified the accuracy of our measurements by comparing with finite-element simulations. The proposed low-cost automatic setup and measuring method were shown to be capable of obtaining accurate measurements of the desired mechanical properties, and it particularly functions robustly well under noisy industrial environments.

\section{Principles}

2.1. Fringe Projection. Fringe projection system, as shown schematically in Figure 1, casts cosine-distributed gray-level fringes onto the membrane surface. The fringe image is then captured by charge-coupled device (CCD) of a camera. The fringe image is distorted on the membrane surface due to its deformation caused by pressurization. The degree of distortion is related to the deformation of the membrane, which we can use to rebuild the $3 \mathrm{D}$ deformation distribution of membrane $[12,13]$. Generally, the intensity distribution of image can be expressed as

$$
I(x, y)=I_{a}(x, y)+I_{b}(x, y) \cos \left(\frac{2 \pi}{P_{0}} x+\Delta \phi(x, y)\right),
$$

where $I_{a}(x, y)$ is average intensity, $I_{b}(x, y)$ is amplitude of modulation strength, $P_{0}$ is period of the projected fringe, and $\Delta \phi(x, y)$ is phase difference due to distortion.

Figure 2 shows the geometric relation among components of the projection system. Setting the unpressurized film surface as the reference plane centered at $O$, the digital light projector (DLP) and CCD are, respectively, located at $O_{p}$ and $\mathrm{O}_{c}$. The line of $\overline{\mathrm{OO}_{c}}$ is normal to the reference plane, and the angle between $\overline{O_{c}}$ and $\overline{O O_{p}}$ is $\theta$. The vertical distances between DLP to the reference plane and CCD to the reference plane are $L_{p}$ and $L_{c}$, respectively. The horizontal distance between DLP and CCD is L. Accordingly, the height of the distorted film $z(x, y)$ can be derived and related to the intensity phase difference distribution of fringe image as [14]

$$
z(x, y)=\frac{P_{0}}{2 \pi \cos \theta} \frac{L_{P}}{L} \Delta \phi(x, y)=k \Delta \phi(x, y),
$$

where the proportional constant $k$ depends solely on geometric setting of the projection system and phase difference distribution $\Delta \phi(x, y)$ can be obtained by a phase retrieval method using four-step phase shifting which will be described in the following section.

2.2. Four-Step Phase Shifting Method. Four-step phase shifting method [15] is applied here for the retrieval of phase information of fringe image cast on a test film. Besides the reference fringe projection, three additional projected fringes with a $\pi / 2$ increment of phase each time are continuously cast on test film. Therefore, four intensity distributions of fringe images can be written as

$$
\begin{gathered}
I_{1}(x, y)=I_{a}(x, y)+I_{b}(x, y) \cos (\phi(x, y)), \\
I_{2}(x, y)=I_{a}(x, y)+I_{b}(x, y) \cos \left(\phi(x, y)+\frac{\pi}{2}\right), \\
I_{3}(x, y)=I_{a}(x, y)+I_{b}(x, y) \cos (\phi(x, y)+\pi), \\
I_{4}(x, y)=I_{a}(x, y)+I_{b}(x, y) \cos \left(\varphi(x, y)+\frac{3 \pi}{2}\right),
\end{gathered}
$$




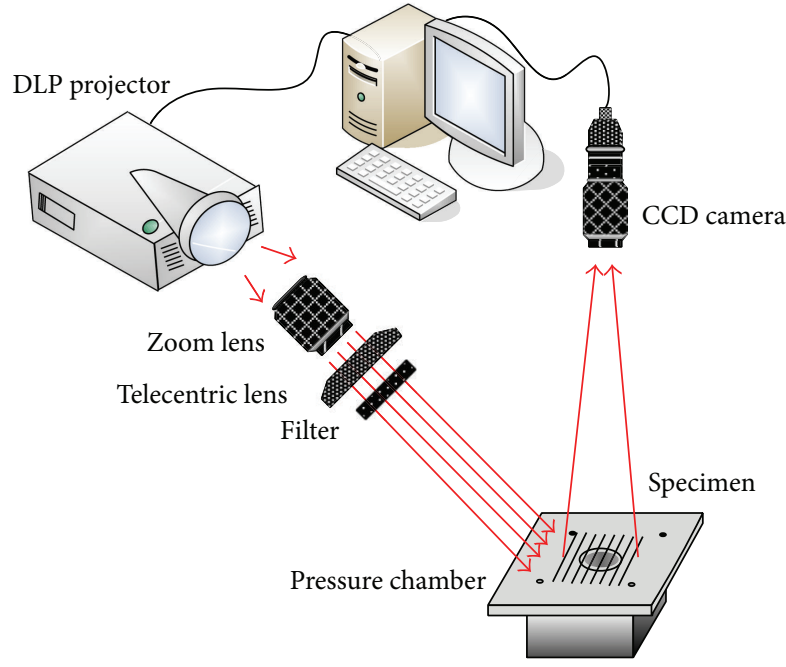

Figure 1: An illustration of fringe projection system.

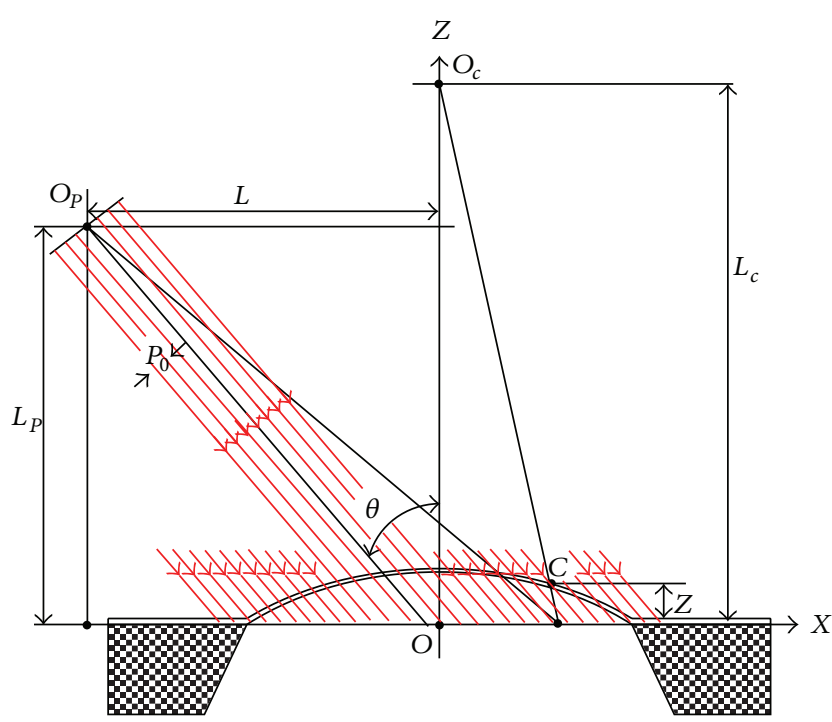

FIgURE 2: Geometric configuration of fringe projection system.

where $I_{a}(x, y)$ and $I_{b}(x, y)$ are defined the same as in (1), and $\Delta \phi(x, y)$ is the phase of image at the position $(x, y)$. From trigonometric identities,

$$
\begin{aligned}
I_{2}(x, y) & =I_{a}(x, y)+I_{b}(x, y) \cos \left(\varphi(x, y)+\frac{\pi}{2}\right) \\
& =I_{a}(x, y)-I_{b}(x, y) \sin (\varphi(x, y)), \\
I_{4}(x, y) & =I_{a}(x, y)+I_{b}(x, y) \cos \left(\varphi(x, y)+\frac{3 \pi}{2}\right) \\
& =I_{a}(x, y)+I_{b}(x, y) \sin (\varphi(x, y)) .
\end{aligned}
$$

Subtracting (8) from (7), we obtain

$$
I_{4}(x, y)-I_{2}(x, y)=2 I_{b}(x, y) \sin (\varphi(x, y)),
$$

and likewise subtracting (5) from (3), we get

$$
I_{1}(x, y)-I_{3}(x, y)=2 I_{b}(x, y) \cos (\varphi(x, y)) .
$$

Further divining (9) by (10), we have

$$
\frac{I_{4}-I_{2}}{I_{1}-I_{3}}=\tan \varphi(x, y)
$$

where phase can be retrieved by

$$
\varphi(x, y)=\tan ^{-1} \frac{I_{4}-I_{2}}{I_{1}-I_{3}} .
$$

Note that there will be a $2 \pi$ discontinuity in the distribution of $\phi(x, y)$ due to the range of arctangent. For this, phase unwrapping is further applied to obtain the continuous distribution of $\phi(x, y)$.

2.3. Bulge Test. Figure 3 is a schematic representation of bulge test. A test film is fixed on the circular opening, with radius $a$, of the gas chamber that is pressurized by a uniform pressure $P$. The deformation of film can be expressed as [16]

$$
w=\sqrt{\left(R^{2}-r^{2}\right)}-\left(R-w_{0}\right)
$$

where $R$ is the radius of curvature of the deforming film, $w$ is the height of the film at radial position $r$, and $w_{0}$ is the height of the film right at the center. Generally speaking $R \gg a \gg$ $w_{0}, \sqrt{\left(R^{2}-r^{2}\right)}$ can therefore be approximated by

$$
\sqrt{\left(R^{2}-r^{2}\right)} \approx R\left(1-\frac{r^{2}}{2 R^{2}}\right),
$$

and (13) can then be simplified to

$$
w \approx R\left(1-\frac{r^{2}}{2 R^{2}}\right)-\left(R-w_{0}\right)=w_{0}-\frac{r^{2}}{2 R} .
$$

Applying Pythagorean theorem to the right triangle $\mathrm{OAB}$ in Figure 3, we can have

$$
\left(R-w_{0}\right)^{2}+a^{2}=R^{2},
$$

or

$$
R=\frac{w_{0}}{2}+\frac{a^{2}}{2 w_{0}} \approx \frac{a^{2}}{2 w_{0}} .
$$

Substituting (17) into (15), we obtain

$$
w=w_{0}-\frac{r^{2}}{2} \frac{2 w_{0}}{a^{2}}=w_{0}\left(1-\frac{r^{2}}{a^{2}}\right),
$$

which basically states that the axis-symmetric deformation distribution with respect to $r$ is parabolic.

According to Timoshenko's assumption [17], the deforming circular film would have no radial displacement at positions of $r=0$ and $r=a$. Therefore, the radial displacement $u$ can be approximated by

$$
u=r(a-r)\left(K_{1}+K_{2} r\right)
$$




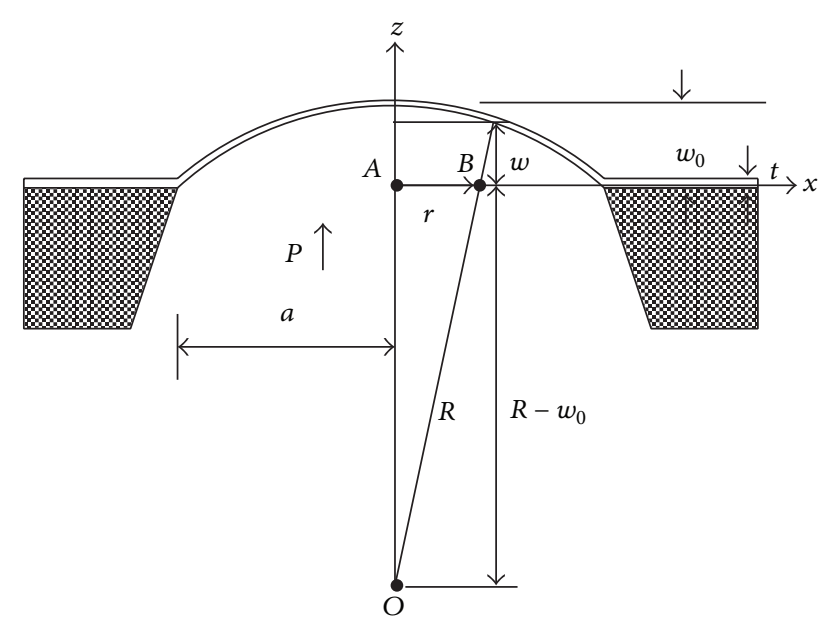

FIGURE 3: Schematic representation of bulge test.

where $K_{1}$ and $K_{2}$ are constants remaining to be determined. Applying Hook's law and considering the residual stress of film, its radial force $N_{r}$ and tangential force $N_{t}$, per unit length, can be related to their counterparts in strain $\varepsilon_{r}$ and $\varepsilon_{t}$ as

$$
\begin{aligned}
& N_{r}=t\left(\sigma_{r}+\sigma_{0}\right)=\frac{E t}{1-v^{2}}\left(\varepsilon_{r}+\nu \varepsilon_{t}\right)+t \sigma_{0} \\
& N_{t}=t\left(\sigma_{t}+\sigma_{0}\right)=\frac{E t}{1-v^{2}}\left(\varepsilon_{t}+\nu \varepsilon_{r}\right)+t \sigma_{0}
\end{aligned}
$$

where $\sigma_{0}$ is residual stress, $t$ is the thickness, $v$ is the Poisson ratio, and $E$ is the Young's modulus of the film. Further, $\varepsilon_{r}$ and $\varepsilon_{t}$ can be related to vertical and radial displacements, $w$ and $u$, as

$$
\begin{gathered}
\varepsilon_{r}=\frac{d u}{d r}+\frac{1}{2}\left(\frac{d w}{d r}\right)^{2}, \\
\varepsilon_{t}=\frac{u}{r}
\end{gathered}
$$

Neglecting the strain energy of bending due to its smallness, the total strain energy $V_{\text {system }}$ of the film would be just the strain energy of stretching $V_{\text {stretching: }}$ :

$$
\begin{aligned}
V_{\text {system }}= & V_{\text {Stretching }}=\frac{1}{2} \int_{0}^{2 \pi} \int_{0}^{a}\left(N_{r} \varepsilon_{r}+N_{t} \varepsilon_{t}\right) r d r d \theta \\
= & \frac{\pi E t}{1-\nu^{2}} \int_{0}^{a}\left(\varepsilon_{r}^{2}+\varepsilon_{t}^{2}+2 \nu \varepsilon_{r} \varepsilon_{t}\right) r d r \\
& +\pi \sigma_{0} t \int_{0}^{a}\left(\varepsilon_{r}+\varepsilon_{t}\right) r d r .
\end{aligned}
$$

$K_{1}$ and $K_{2}$ can be decided from energy variation principle, and $V_{\text {system }}$ can be further derived to depend on maximum deformation $w_{0}$ :

$$
V_{\text {system }}=\frac{\pi E t w_{0}^{4}(7-v)}{24 a^{2}(1-\nu)}+\pi \sigma_{0} t w_{0}^{2} .
$$

By principle of least action, for a given virtual displacement $\delta w_{0}$, the corresponding virtual work would be equal to $\delta V_{\text {system: }}$

$$
\delta V_{\text {system }}=\frac{d V_{\text {system }}}{d w_{0}} \delta w_{0}=\int_{o}^{2 \pi} \int_{0}^{a} P\left(\delta w_{0}\right) r d r d \theta,
$$

from which

$$
P=\frac{2 \delta V_{\text {system }}}{\pi a^{2} \delta w_{0}}=4 \frac{t \sigma_{0}}{a^{2}} w_{0}+\left(\frac{7-v}{3}\right)\left(\frac{E}{1-v}\right) \frac{t}{a^{4}} w_{0}^{3} .
$$

Equation (27) means that once we obtain the data of $P$ (pressure applied to film) versus $w_{0}$, we can do curve fitting to obtain the coefficients of the cubic polynomial in (27). With given $t, a$, and $E$, we can find out the Poisson ratio $v$ from these coefficients. Biaxial modulus $M=E /(1-\nu)$ can then be found out too.

\section{System Configuration}

The system of bulge test is shown in Figure 4, which includes three subsystems: optical, pressure loading, and control subsystems. Each subsystem is described in the following.

3.1. Optical System. The optical system consists of fringe projection part and image capturing part. The fringe projection system is composed of a digital light projector (PJ503D, ViewSonic Co., Ltd., Japan), a zoom lens (AF Micro-Nikkor $60 \mathrm{~mm} \mathrm{f/2.8} \mathrm{D,} \mathrm{Nikon} \mathrm{Co.,} \mathrm{Ltd.,} \mathrm{Japan),} \mathrm{a} \mathrm{teleconverter} \mathrm{lens}$ (TC-E2, Nikon Co., Ltd., Japan), and a neutral density filter (ND8 Filter $52 \mathrm{~mm}$, Kenko Co., Ltd., Japan). The digital light projector (with its built-in fisheye lens replaced by a zoom lens) projects cosine-distributed gray-level fringes, amplified by the telecentric lens [18], onto the test film and then the deformed fringe image is reflected and captured, through a zoom lens (GMZ18108, Goyo Optical Inc., Japan), by a 256 RGB full color CCD (scA640-74fc, Basler Co., Ltd., German) with a resolution of $640 \times 320$ pixels. The fringe period was set to $0.5624 \mathrm{~mm}$ and projection angle $\theta$ (as shown in Figure 2) was $25^{\circ}$.

As shown in Figure 2, the test film was fixed onto an aluminum plate that is used as the reference plane. The CCD was mounted right above the reference plane. The RGB image captured by CCD was further reduced to a $256 \times 256$ image with its field of view being $18.56 \mathrm{~mm}$ and centered at pixel coordinate $(320,160)$. Using the formula below, we can transform this color image into a gray-level image that is ready for further image processing [19]:

$$
\text { Gray }=0.2989 \times R+0.5870 \times G+0.1140 \times B \text {. }
$$

3.2. Loading System. The pressure loading system, shown in Figure 5, used high-pressure nitrogen as the pressure source, which is reduced to $150 \mathrm{KPa}$ under room temperature through pressure regulator. The test film was fixed to the top opening of a circular aluminum-made pressure chamber of $15 \mathrm{~mm}$ in diameter. White paint was particularly sprayed onto the test film to increase its reflectivity. A large-volume 


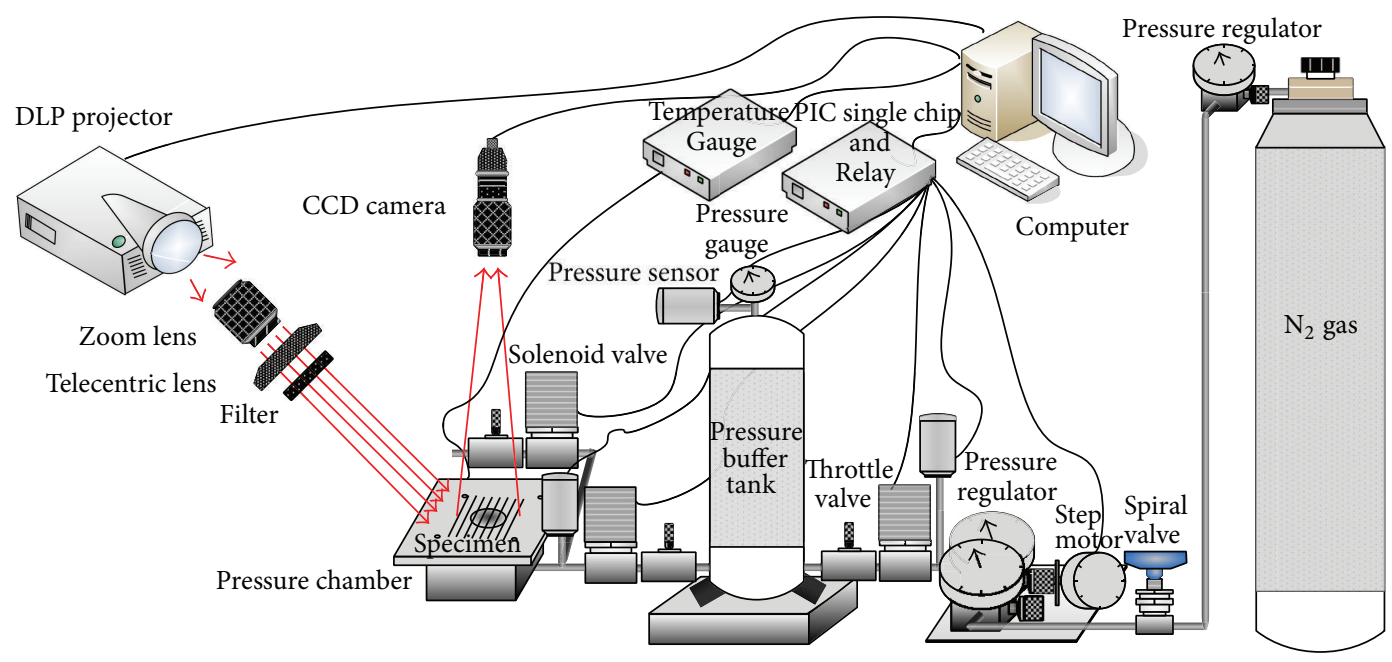

FIGURE 4: System configuration of bulge test including three subsystems: optical, pressure loading, and control subsystems.

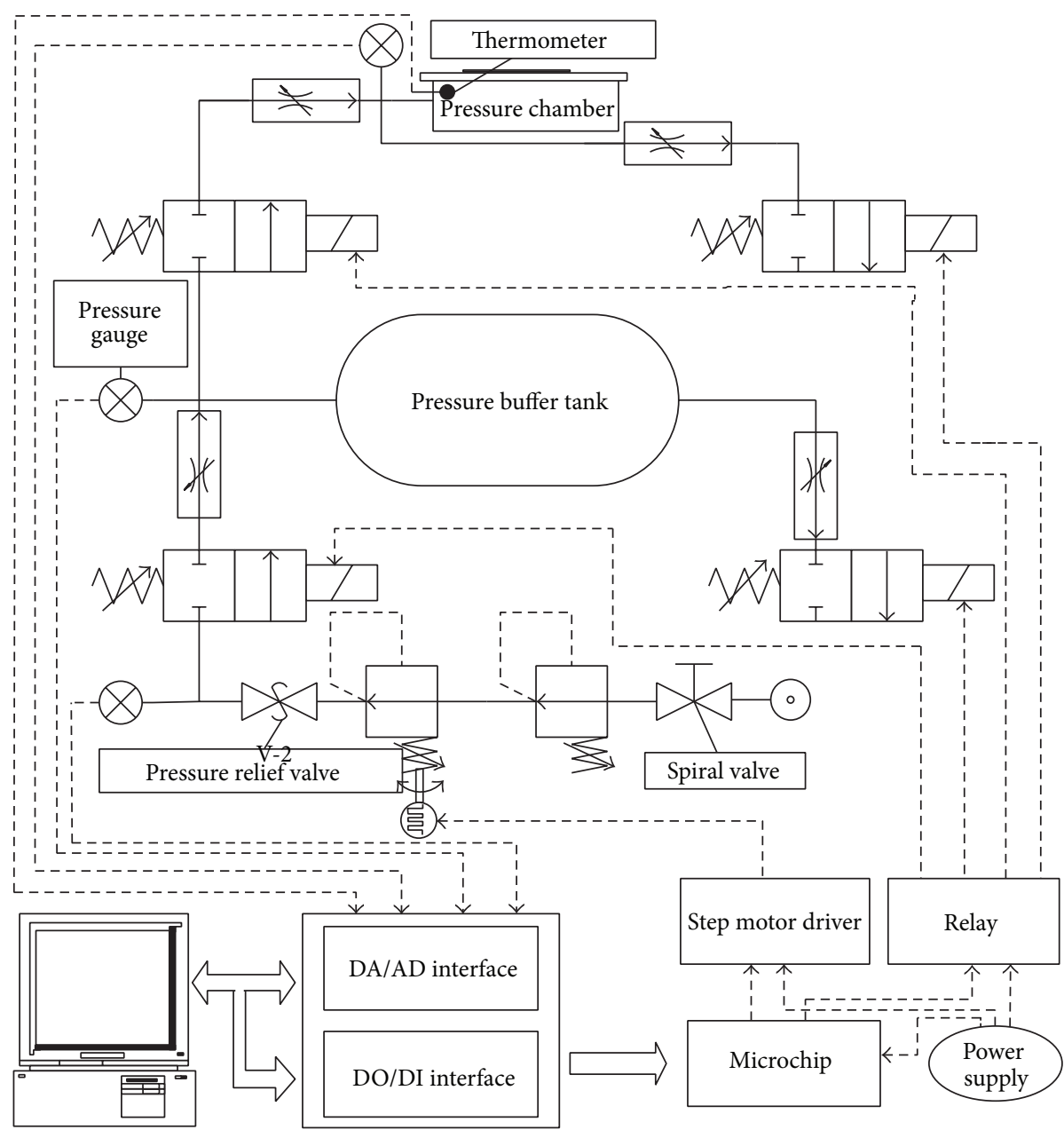

FIGURE 5: A control block diagram of pressure loading system. 


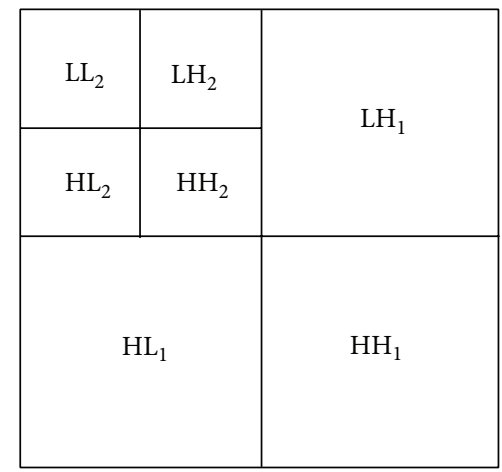

FIGURE 6: A schematic presentation of multiwavelet transformation.

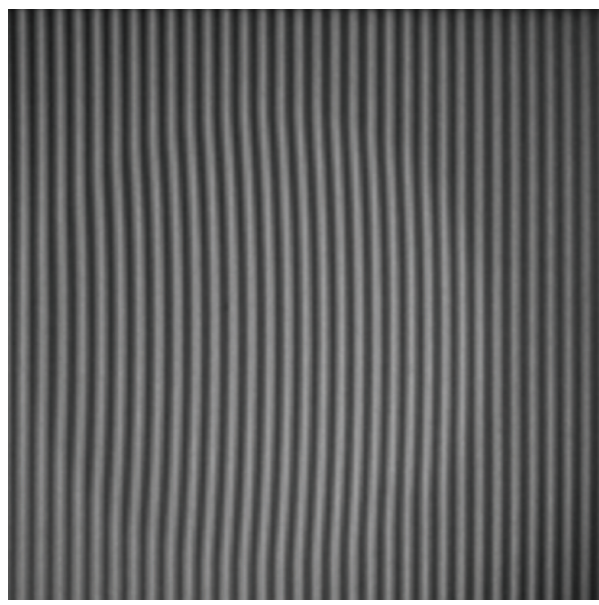

(a)

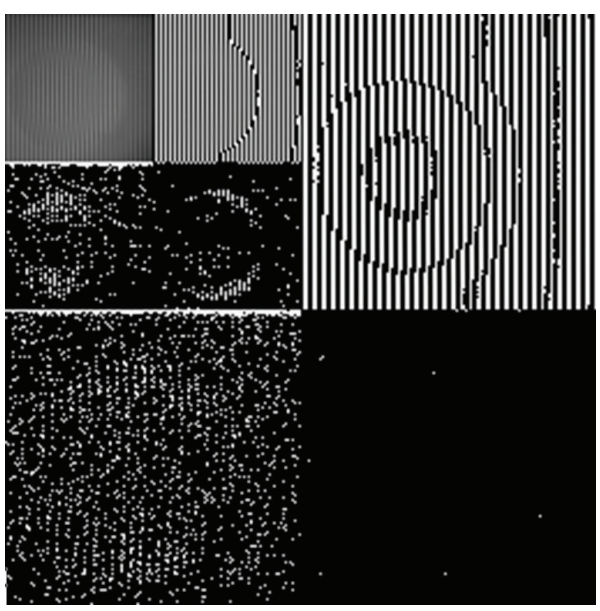

(c)

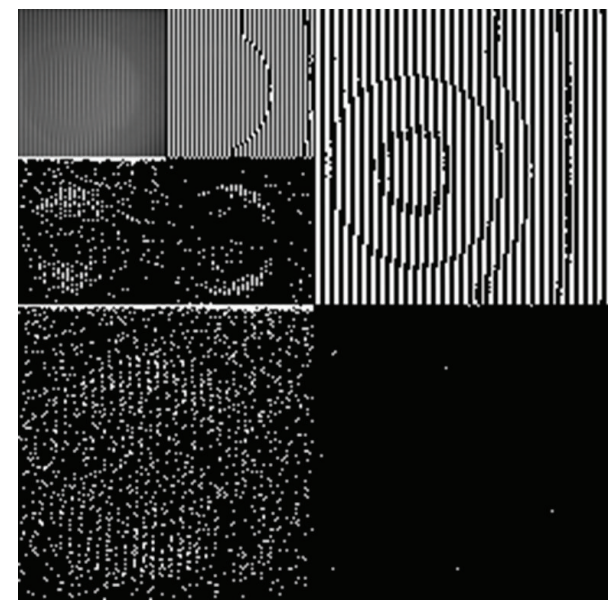

(b)

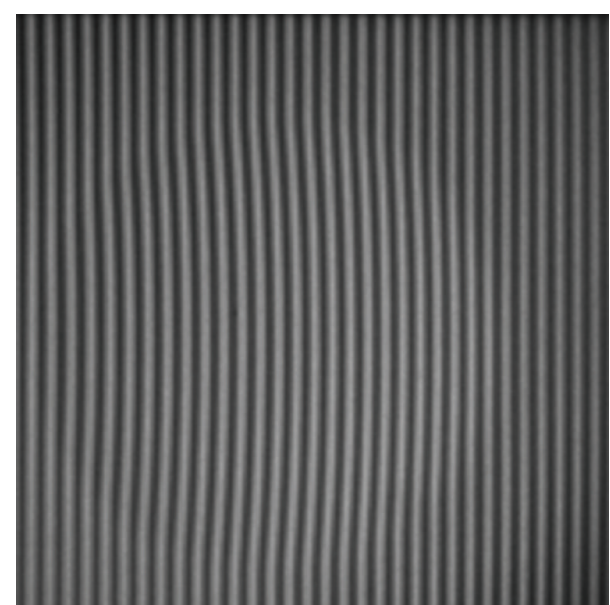

(d)

FIGURE 7: Denoising via Haar-wavelet transformations: (a) original image; (b) decomposed images using wavelet transformation before denoising; (c) decomposed images after denoising; (d) image reconstruction after denoising.

buffer tank was installed between the gas chamber and the pressure source, connected to them via a pneumatic solenoid valve and a pressure sensor (see Figure 5). The pressure sensor (AST4000AV15P4E0, American Sensor Technologies, Inc., American) has a working pressure range of $-101.35 \sim$ $103.42 \mathrm{KPa}$ with an accuracy of $\pm 0.5 \%$ BFSL (Best Fit Straight
Line, BFSL). The volume of the pressure buffer tank used here is 12.00 liters, and the volume of the gas chamber is 0.05 liters approximately. Since the volume of the pressure buffer tank is much larger than that of gas chamber, the pressure of the gas chamber would be almost the same as that of pressure buffer tank by Boyle's law once their connection is on. When 


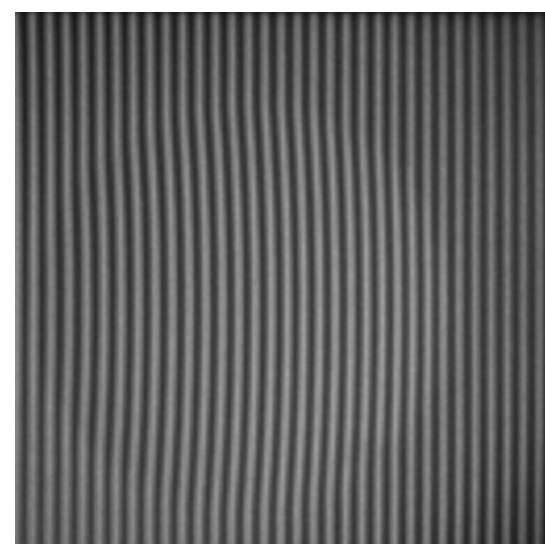

(a)

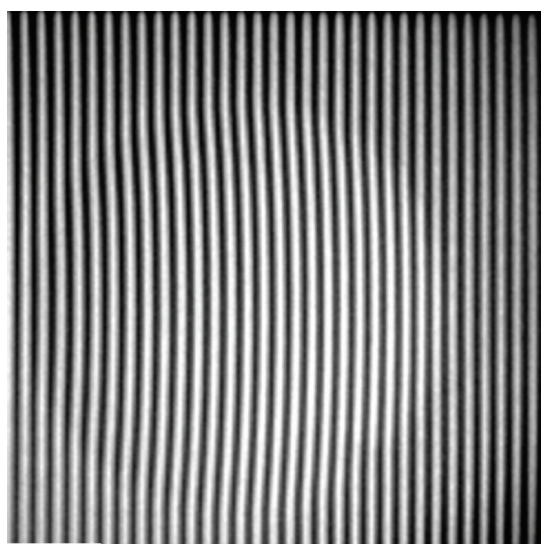

(c)

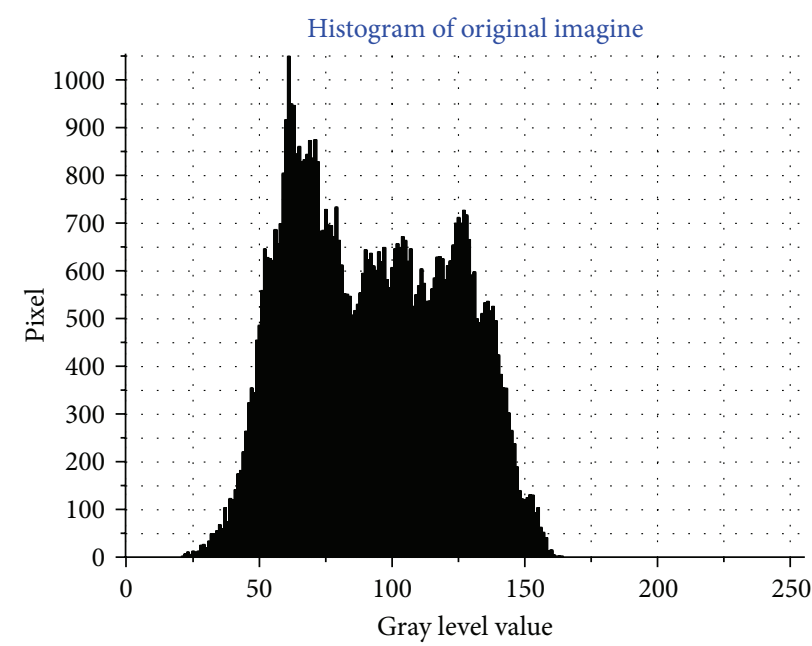

(b)

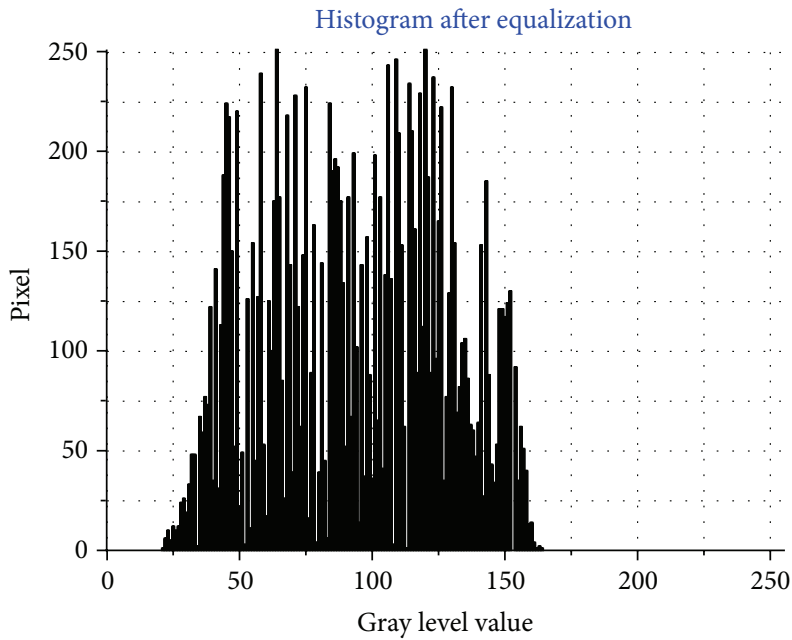

(d)

FIGURE 8: Fringe image before and after histogram equalization: (a) original image; (b) histogram of original image; (c) enhanced image after histogram equalization; (d) histogram of enhanced image.

applying pressure, the solenoid valve between pressure source and pressure buffer tank is opened, while the one between pressure buffer tank and gas chamber is closed. This is to avoid a direct pressure impact onto the test film. After the desired pressure in the buffer tank is reached and kept steady for a while, the solenoid valve between pressure buffer tank and test chamber is then opened to exert the designated pressure onto the test film.

3.3. Control System. The current control system uses a personal computer (with a $\mathrm{C}++$ program) and PIC controller (connected to the RS232 port of computer) to actuate the solenoid valve. The pressure is regulated by a feedback control through a pressure-sensing circuit connected to an analogdigital converter card (PCI-1802, ICP DAS Co., Ltd., Taiwan) mounted on the computer. The pressure-sensing circuit consists of a pressure sensor and a $0.5 \mathrm{~K} \Omega$ resistor connected in series. The pressure sensor measures the pressure and output an electric current with a range of $0 \sim 20 \mathrm{~mA}$, and this output current further transforms to a voltage signal with the range $0 \sim 10 \mathrm{~V}$ when passing through the resistor. The voltage signal serves as the input into analog-digital converter card that measures the pressure and records it into the computer with the relation between pressure and voltage calibrated to be

$$
P=(V-6.330) \times 25.876+1.310
$$

where $P$ is the pressure (in $\mathrm{kPa}$ ) and $V$ is the voltage (in volt). As to the PIC controller, the signal from RS232 of computer is $5 \mathrm{~V}$ voltage signal that is output to a single-chip controller (Microchip PIC16F877). This controller further outputs signals to drive the stepper motor (Oriental motor Co., Ltd, Japan) and control solenoid valves. The stepper motor connects to the pressure-regulating valve, and it drives the valve through a pair of gears with gear ratio being 3.55 . 


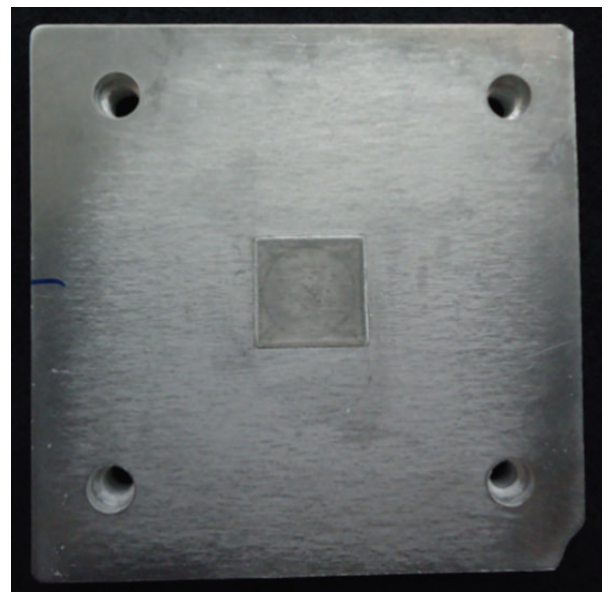

(a)

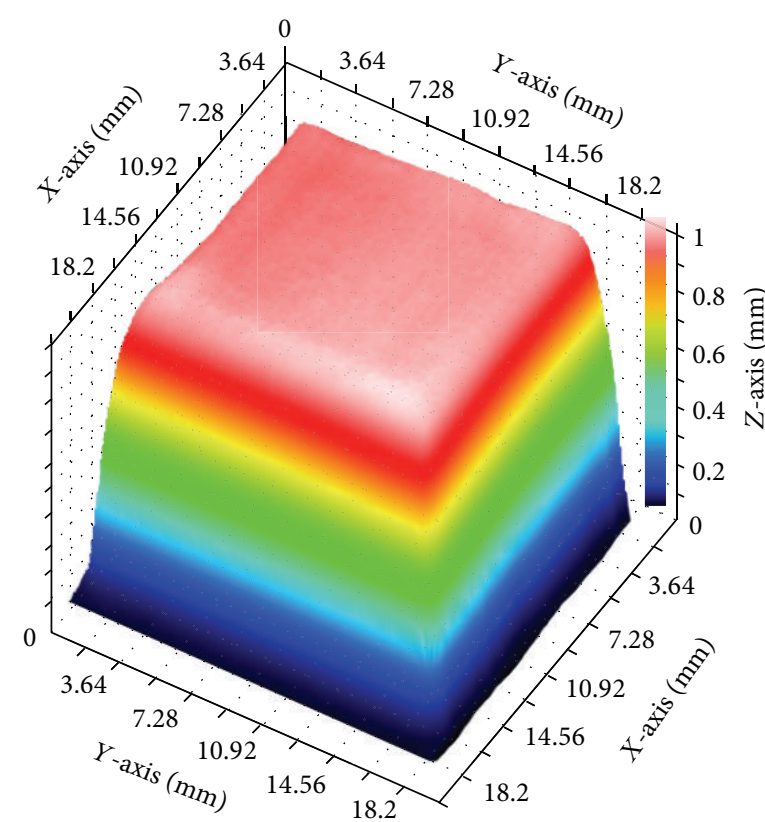

(b)

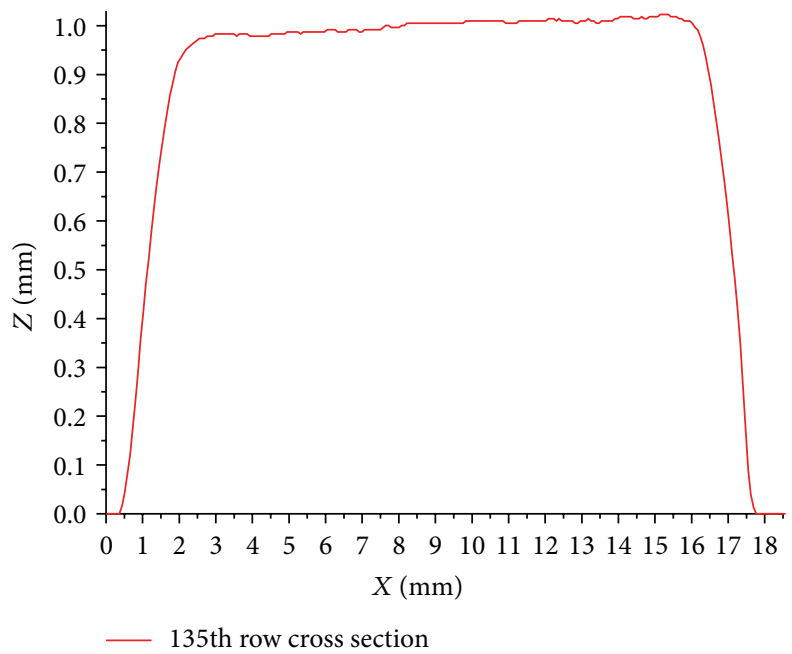

(c)

FIGURE 9: Calibration with an acrylic trapezoid with the height of $1.000 \mathrm{~mm}$ : (a) acrylic trapezoid located at the center of testing plate; (b) the measured 3D surface of trapezoid; (c) the measured height distribution on the $x-x$ cross-section plane passing through the center of trapezoid.

\section{Image Processing Methods}

Here we used Haar wavelet to denoise the fringe image. The basic idea of the wavelet transform is to decompose a signal into different scales and resolution. The fringe image after first Haar-wavelet transformation would be decomposed into four subbands: $\mathrm{LL}_{1}, \mathrm{LH}_{1}, \mathrm{HL}_{1}$, and $\mathrm{HH}_{1}$, where the first $\mathrm{L}$ (or $\mathrm{H})$ denotes the low-frequency (or high-frequency) mode in $x$ direction, while the second $\mathrm{L}$ ( or $\mathrm{H}$ ) denotes the same as above but in $y$ direction. 1 means the first transformation. We then again applied Haar-wavelet transformation to $\mathrm{LL}_{1}$ and further decomposed $\mathrm{LL}_{1}$ into $\mathrm{LL}_{2}, \mathrm{LH}_{2}, \mathrm{HL}_{2}$, and $\mathrm{HH}_{2}$ as illustrated in Figure 6.
When doing denoising, all subbands in Figure 6 except $\mathrm{LL}_{2}$ will be filtered with a Donoho frequency threshold expressed as follows [20, 21]:

$$
\delta=s \sqrt{2 \log N}
$$

where $s$ is the standard deviation of the gray-scale image and $N$ is the number of pixels of image.

Denoising can effectively eliminate unwanted periodic patterns and render homogeneous illumination on the fringe image. The denoising process of the current fringe image using 2D Haar-wavelet transform and inverse transform is 


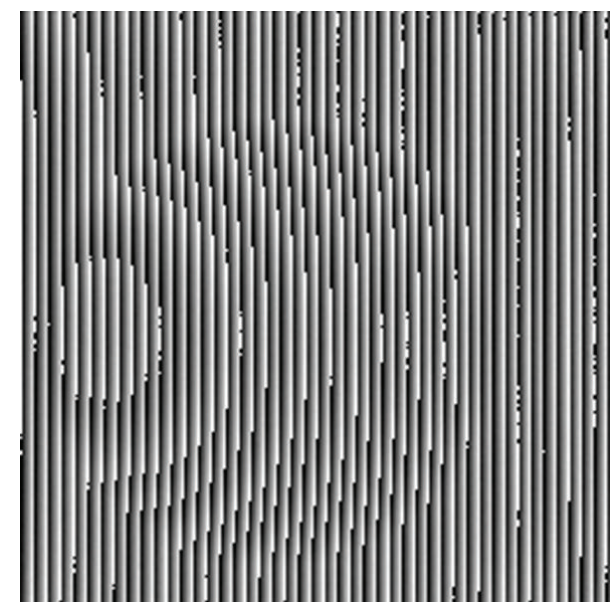

(a)

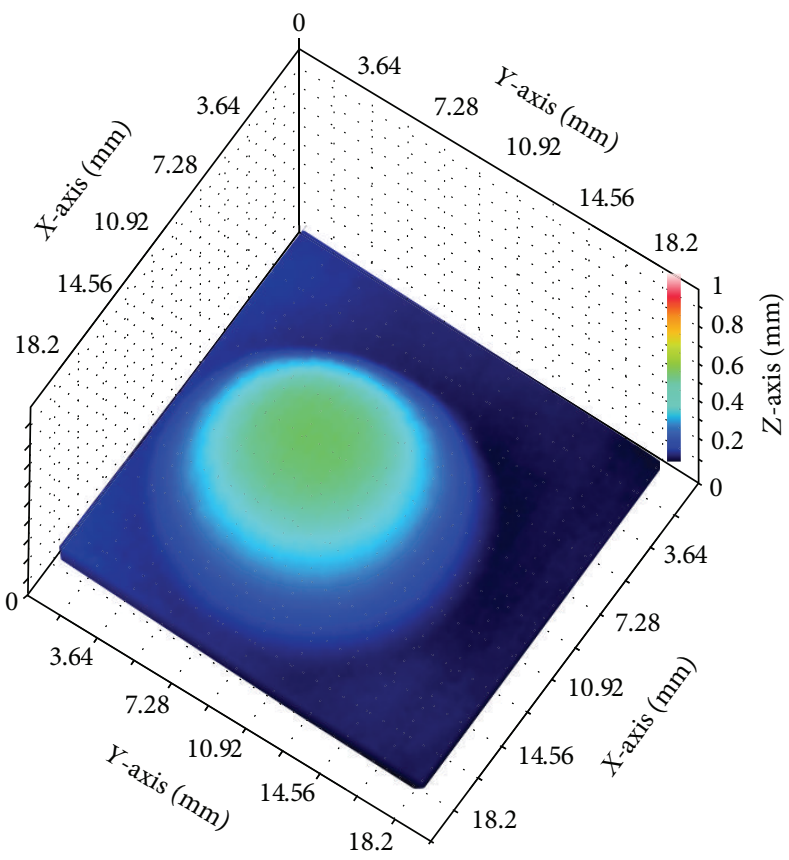

(c)

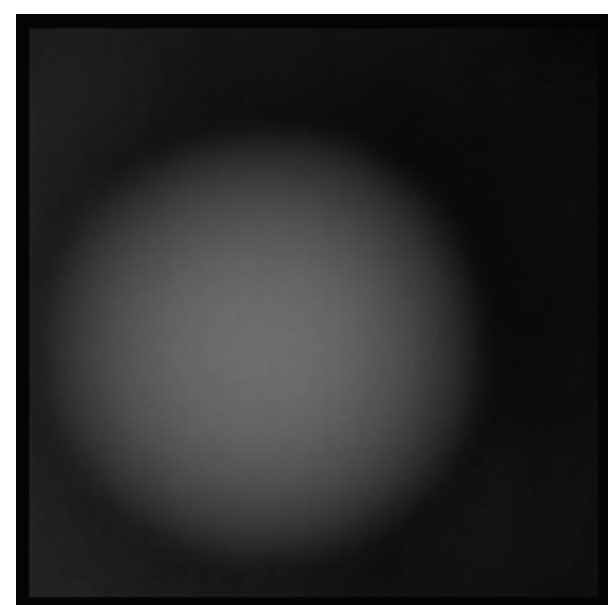

(b)

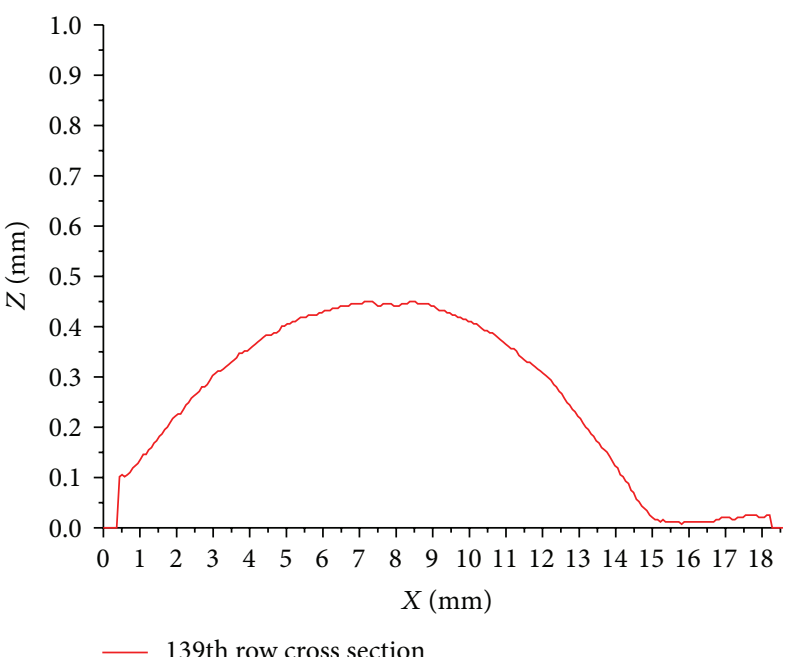

(d)

FIGURE 10: 3D surface construction of test film: (a) phase distribution before phase unwrapping and extension; (b) phase distribution after phase unwrapping and extension; (c) constructed 3D surface of the test film; (d) the deformation distribution on a cross-section plane, parallel to $y z$-plane and through the axisymmetric center, of 3D surface.

shown in Figure 7. Due to highly accurate optical measurements, one might notice that the difference between images of Figures 7(a) and 7(d), noted as before and after denoising, is actually minute.

Besides denoising, we need to enhance the contrast of image, which would help to retrieve the phase more accurately. Here, we used histogram equalization to do the contrast enhancement $[22,23]$. First, we obtained the gray scale distribution in histogram of the fringe image as shown in Figures 8(a) and 8(b) and then adjusted the gray scale of each pixel by the following formula:

$$
u_{k}=\operatorname{int}\left((L-1) \sum_{i=0}^{k} \frac{N_{k}}{N}\right), \quad k=0,1, \ldots, L-1,
$$

where $k$ is the shifted gray scale; $N_{k}$ is the number of pixels in shifted gray scale $k$; $N$ is the total number of pixels; int(.) denotes the integer function by rounding; $u_{k}$ is the adjusted gray scale after equalization for pixels originally in shifted gray scale $k$. The meaning of shifted gray scale is that if $i=$ $m, m+1, \ldots, n$ is the gray scale with nonzero population, then $k=i-m$, with $L=n-m+1$. The fringe image after adjustment would have better contrast as shown in Figures 8(c) and 8(d).

\section{Results and Discussions}

5.1. Calibration. Before conducting measurements, the system needs to be calibrated to obtain the coefficient $k$ in (2) [24]. Here we used an acrylic trapezoid with the height known 


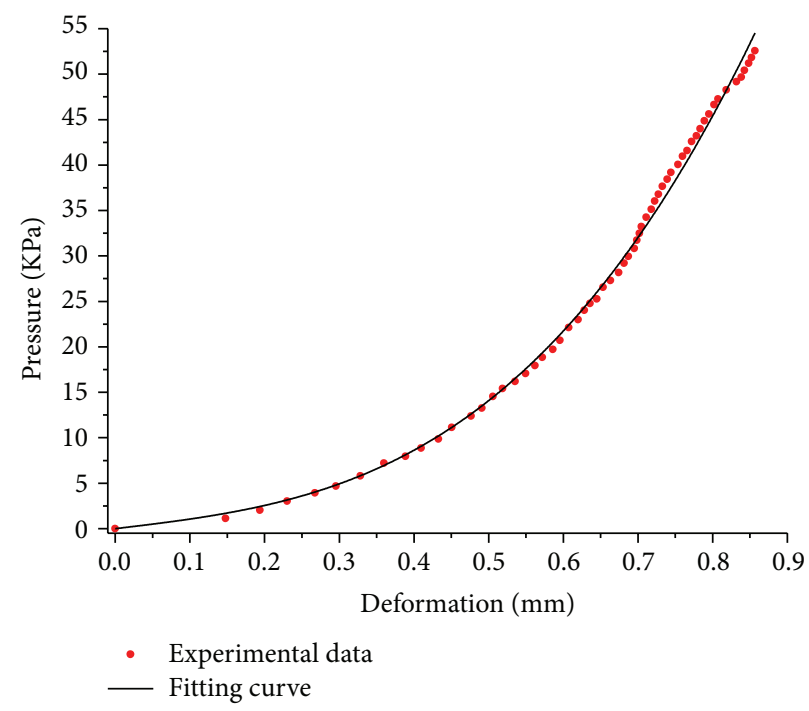

FIGURE 11: The measurements of pressure versus maximum film deformation and its 3rd-order-polynomial least-square fit.

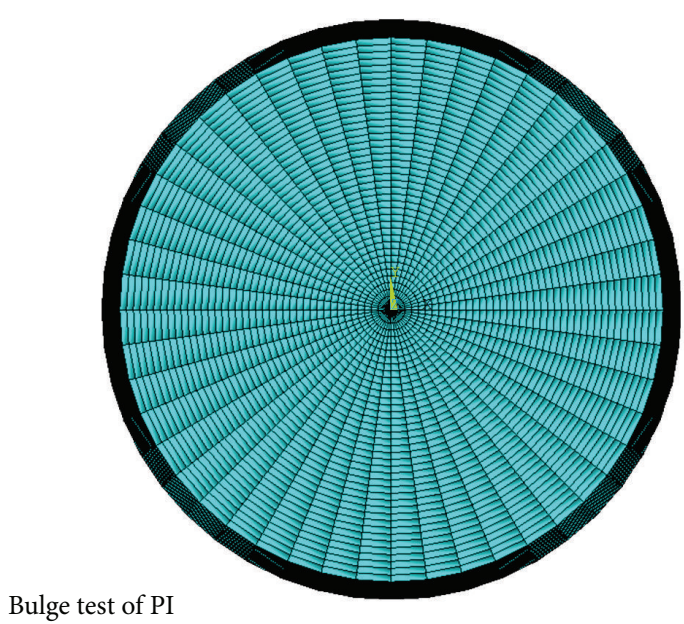

Bulge test of PI

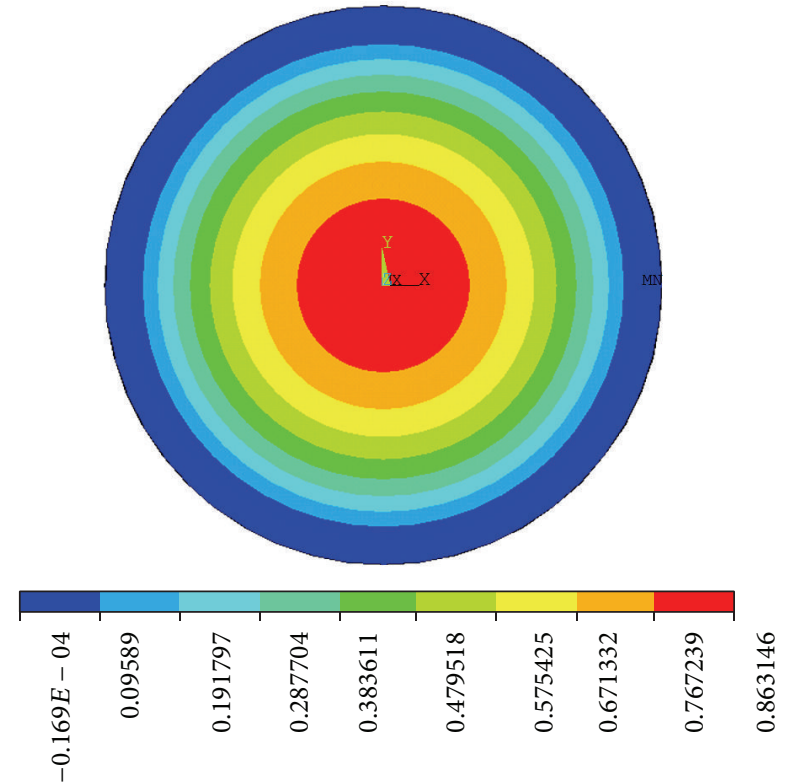

Bulge test of PI

(a)

(b)

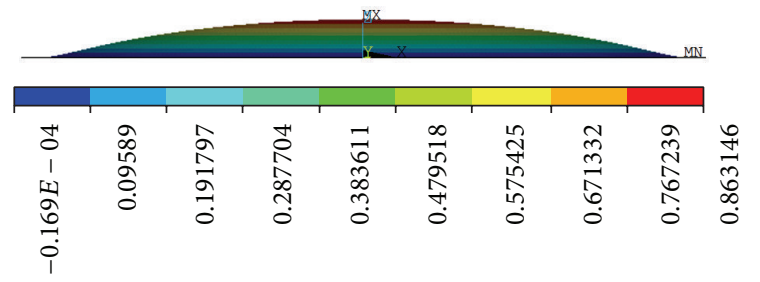

Bulge test of PI

(c)

FIGURE 12: ANSYS finite element analysis: (a) mesh plot of the circular membrane; (b) an example of the deformation distribution in top view; (c) the side-view counterpart of (b). 


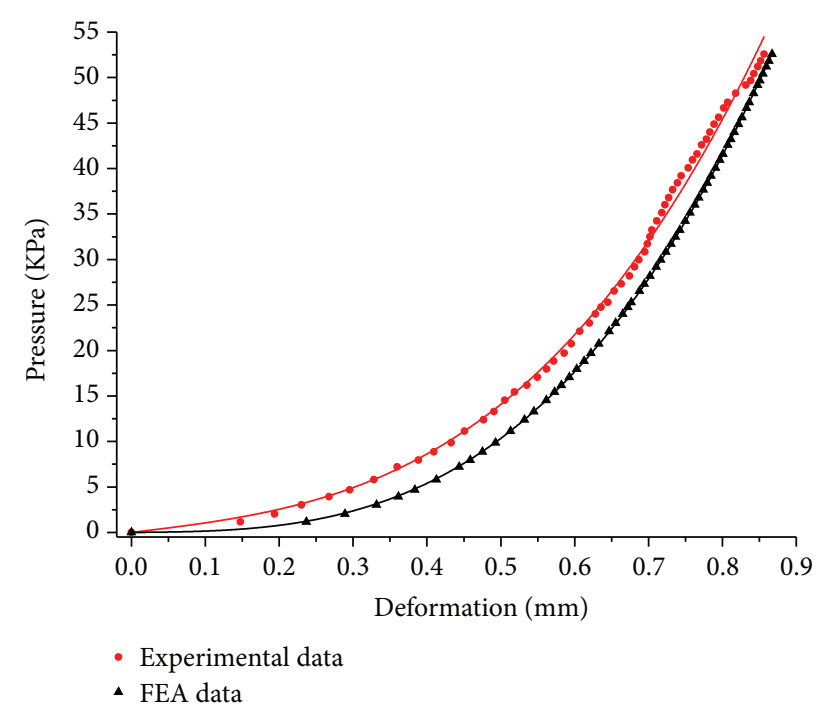

FIGURE 13: Comparison of measurements with a finite element simulation. The error between them is within $8 \%$.

to be $1.000 \mathrm{~mm}$ as shown in Figure 9 to do the calibration. The value of $k$ is obtained to be $0.15647 \pm 0.00218 \mathrm{~mm} / \mathrm{rad}$ with the height of trapezoid being measured to be $1.00000 \pm$ $0.00087 \mathrm{~mm}$.

5.2. Polyimide Film Measurement. From (12), the computed phase angle is always between $-\pi$ and $\pi$, as shown in Figure 10(a). To be able to compute the deformation by (2), we need to unwrap and extend the phase distribution beyond $[-\pi, \pi]$ as shown in Figure 10(b). Finally the computed film deformation distribution is shown in Figure 10(c) with a cross-section of it parallel to $y z$-plane and through the axisymmetric center shown in Figure 10(d).

Here we used polyimide (PI) films with thickness of $25 \mu \mathrm{m}$ (manufactured by PERM TOP Co. Ltd., Taiwan) for measurement experiments. The PI films were pressurized substantially from $0 \mathrm{KPa}$ to $52 \mathrm{KPa}$ with an interval of $1 \mathrm{KPa}$. The maximum pressure was set to be $52 \mathrm{KPa}$, since films tend to rupture if the pressure is larger than $52.57345 \pm$ $0.02194 \mathrm{KPa}$. The measured maximum membrane deformation versus pressure, together with its 3rd-order-polynomial least-square fitting, is shown in Figure 11. The fitted 3rd-order polynomial is

$$
\begin{aligned}
P= & (9.83501 \pm 0.65178) \times w_{0} \\
& +(73.33203 \pm 1.20469) \times w_{0}{ }^{3},(\mathrm{KPa}-\mathrm{mm}),
\end{aligned}
$$

where $P$ is the measured pressure and $w_{0}$ is the measured maximum film deformation. Young's modulus of the PI film was a priori measured to be $2.830 \pm 0.025 \mathrm{GPa}$ by the microtensile test. Applying (27), we can compute the Poisson ratio to be $0.32116 \pm 0.00528$ and the residual stress to be $5.53219 \pm 0.36668 \mathrm{MPa}$. Biaxial modulus can then be computed to be $4.168876 \pm 0.06850 \mathrm{GPa}$.
5.3. Comparison with FEA. In this study, ANSYS finite element analysis is used to verify the accuracy of our measurements. A circular plate was divided into 17,283 elements as shown in Figure 12(a). Applying various pressures to this finite element model, with the inputs of residual stress, Young's modulus and Poisson ratio from our measurements, a series of computational data of maximum deformation versus pressure was obtained with an example of deformation distribution shown in Figures 12(b) and 12(c). The finite-element simulation result agrees very well with our measurements as shown in Figure 13. The error between them is within 8\%. The figure also hints that the agreement would even be better if the pressure applied is larger. However, we did not pursue that, since pressure too large might rupture the film.

\section{Conclusions}

This paper introduced a real-time automatic setup for bulge test, which is integrated with fringe projection to measure the Poisson ration, biaxial modulus, and residual stress of a PI film. The accuracy of measurements was further verified by a finite element analysis. The results have concluded the feasibility of the proposed method. Besides the high accuracy, low cost of setup and endurance to environmental disturbances are also the merits of our apparatus. Generally speaking, it is suitable for all PI film's measuring task either in a laboratory or manufacturing factory.

\section{Conflict of Interests}

The authors declare that there is no conflict of interests regarding the publication of this paper.

\section{Acknowledgments}

This work is in memory of Professor Lu-Ping Chao, a late professor of the Department of Mechanical and ComputerAided Engineering, Feng Chia University. He was also the Ph.D. adviser of one of the authors, Ching-Sen Lin. Also, all the authors would like to thank the support of National Science Council of Taiwan under Grants NSC 100-2221-E035-033 and NSC 100-2632-E-035-001-MY3.

\section{References}

[1] M. C. Choi, Y. Kim, and C. S. Ha, "Polymers for flexible displays: from material selection to device applications," Progress in Polymer Science, vol. 33, no. 6, pp. 581-630, 2008.

[2] H. Zhang, M. D. Poliks, and B. Sammakia, "A roll-to-roll photolithography process for establishing accurate multilayer registration on large area flexible films," Journal of Display Technology, vol. 6, no. 11, pp. 571-578, 2010.

[3] C. L. Tien, T. W. Lin, S. S. Jyu, H. D. Tseng, C. S. Lin, and M. C. Liu, "The measurement of anisotropic stress in obliquelydeposited thin films by fast fourier transform and gaussian filter," Physics Procedia, vol. 19, pp. 21-26, 2011.

[4] M. Iguchi and J. E. Luco, "Vibration of flexible plate on viscoelastic medium," Journal of the Engineering Mechanics Division, vol. 108, no. 6, pp. 1103-1120, 1982. 
[5] B. J. Briscoe, L. Fiori, and E. Pelillo, "Nano-indentation of polymeric surfaces," Journal of Physics D: Applied Physics, vol. 31, no. 19, pp. 2395-2405, 1998.

[6] E. Gacoin, A. Chateauminois, and C. Fretigny, "Measurements of the viscoelastic moduli of an acrylate polymer in bulk and film form using a contact method," Polymer, vol. 45, no. 11, pp. 3789-3796, 2004.

[7] G. M. Odegard, T. S. Gates, and H. M. Herring, "Characterization of viscoelastic properties of polymeric materials through nanoindentation," Experimental Mechanics, vol. 45, no. 2, pp. 130-136, 2005.

[8] B. Schoeberle, M. Wendlandt, and C. Hierold, "Long-term creep behavior of SU-8 membranes: application of the timestress superposition principle to determine the master creep compliance curve," Sensors and Actuators A: Physical, vol. 142, no. 1, pp. 242-249, 2008.

[9] M. P. Orthner, L. W. Rieth, and F. Solzbacher, "High speed wafer scale bulge testing for the determination of thin film mechanical properties," Review of Scientific Instruments, vol. 81, no. 5, Article ID 055111, 2010.

[10] F. Zhu, D. Lei, and X. He, "Measurement system of dynamic microscopic deformation for membrane based on fringe projection," Optical Engineering, vol. 52, no. 3, Article ID 033604, 2013.

[11] B. E. Alaca, K. B. Toga, O. Akar, and T. Akin, "Strain-controlled bulge test," Journal of Materials Research, vol. 23, no. 12, pp. 3295-3302, 2008.

[12] C. Quan, C. J. Tay, X. Y. He, X. Kang, and H. M. Shang, "Microscopic surface contouring by fringe projection method," Optics and Laser Technology, vol. 34, no. 7, pp. 547-552, 2002.

[13] K. Körner, R. Windecker, M. Fleischer, and H. J. Tiziani, "Onegrating projection for absolute three-dimensional profiling," Optical Engineering, vol. 40, no. 8, pp. 1653-1660, 2001.

[14] Z. Zhang, D. Zhang, and X. Peng, "Performance analysis of a 3D full-field sensor based on fringe projection," Optics and Lasers in Engineering, vol. 42, no. 3, pp. 341-353, 2004.

[15] J. C. Wyant, C. L. Koliopoulos, B. Bhushan, and O. E. George, "An optical profilometer for surface characterization of magnetic media," ASLE Transactions, vol. 27, no. 2, pp. 101-113, 1984.

[16] P. Lin, The in-situ measurement of mechanical properties of multi layercoatings [Ph.D. thesis], Massachusetts Institute of Technology, Cambridge, Mass, USA, 1990.

[17] S. P. Timoshenko and S. W. Krieger, Theory of Plates and Shells, McGraw-Hill, New York, NY, USA, 2nd edition, 1959.

[18] F. Zhu, W. Liu, H. Shi, and X. He, "Accurate 3D measurement system and calibration for speckle projection method," Optics and Lasers in Engineering, vol. 48, no. 11, pp. 1132-1139, 2010.

[19] "Convert RGB image or colormap to grayscale," 2013, http://www.mathworks.com/help/images/ref/rgb2gray.html.

[20] D. L. Donoho and J. M. Johnstone, "Ideal spatial adaptation by wavelet shrinkage," Biometrika, vol. 81, no. 3, pp. 425-455, 1994.

[21] D. L. Donoho, "De-noising by soft-thresholding," IEEE Transactions on Information Theory, vol. 41, no. 3, pp. 613-627, 1995.

[22] Y. T. Kim, "Contrast enhancement using brightness preserving Bi-histogram equalization," IEEE Transactions Consumer Electronics, vol. 43, no. 1, pp. 1-8, 1997.

[23] T. K. Kim, J. K. Paik, and B. S. Kang, "Contrast enhancement system using spatially adaptive histogram equalization with temporal filtering," IEEE Transactions on Consumer Electronics, vol. 44 , no. 1 , pp. 82-87, 1998.
[24] S. Zhang and P. S. Huang, "Novel method for structured light system calibration," Optical Engineering, vol. 45, no. 8, Article ID 083601, 2006. 

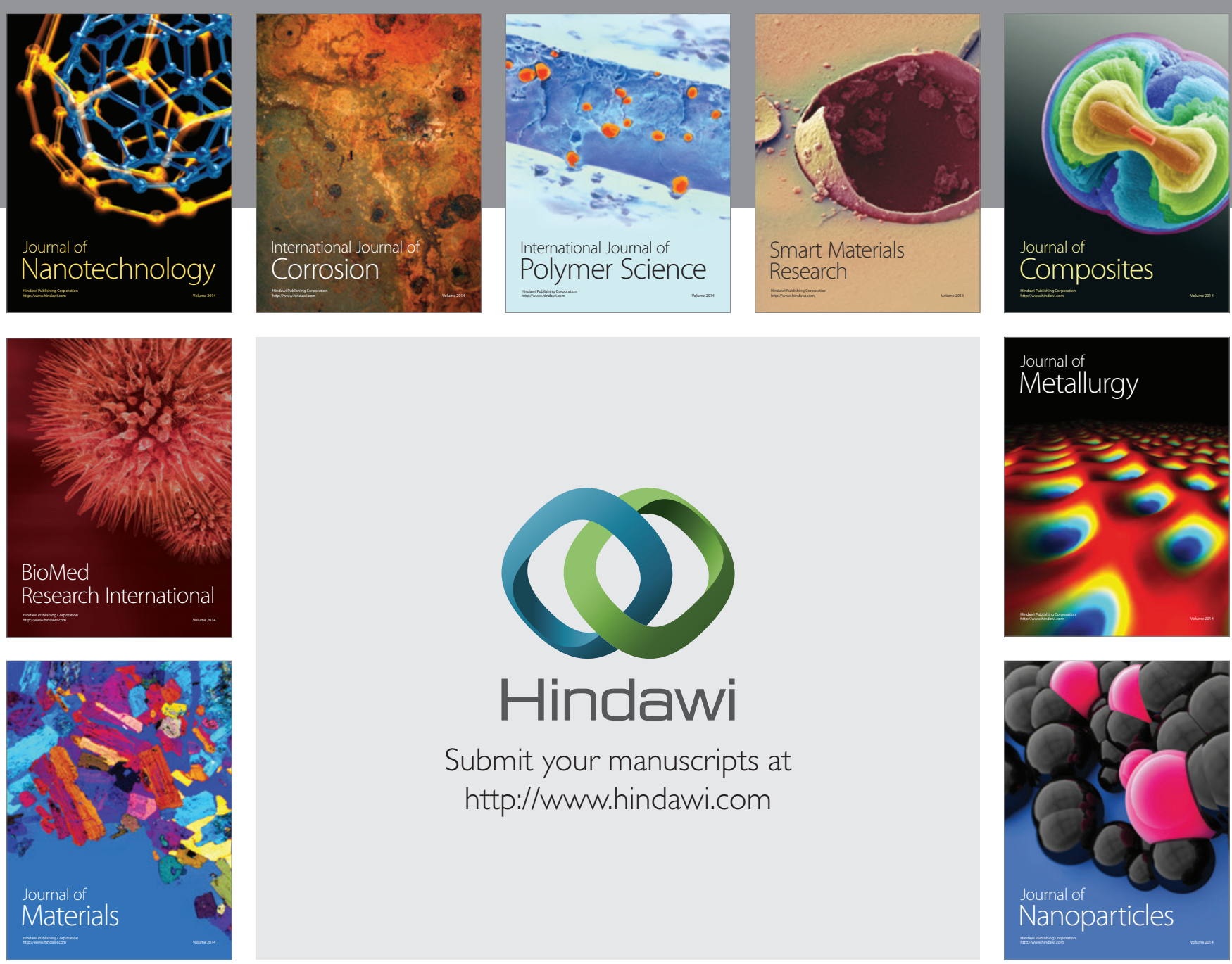

Submit your manuscripts at http://www.hindawi.com
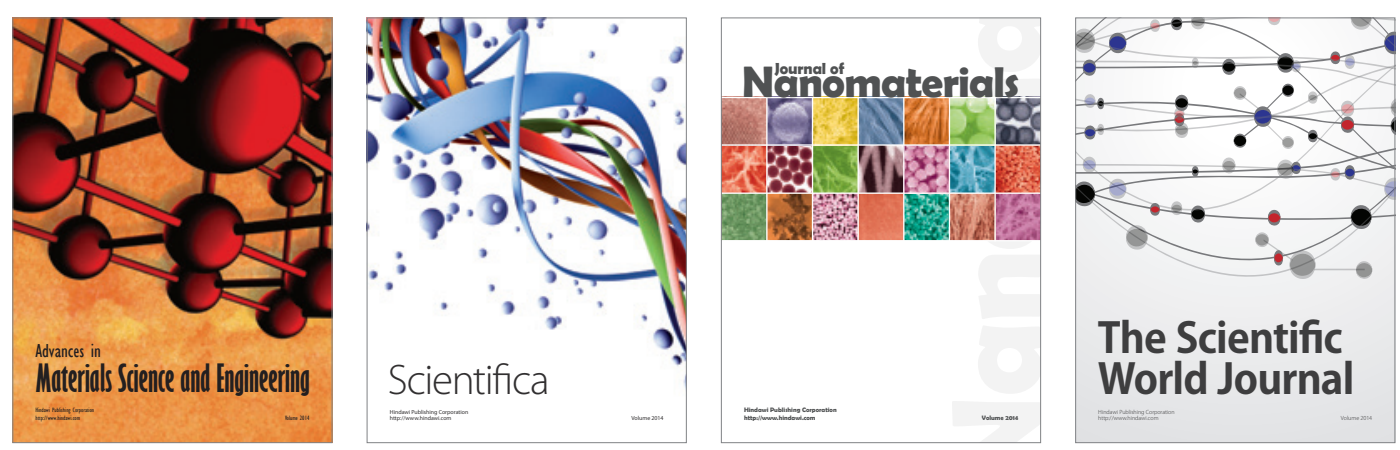

\section{The Scientific World Journal}
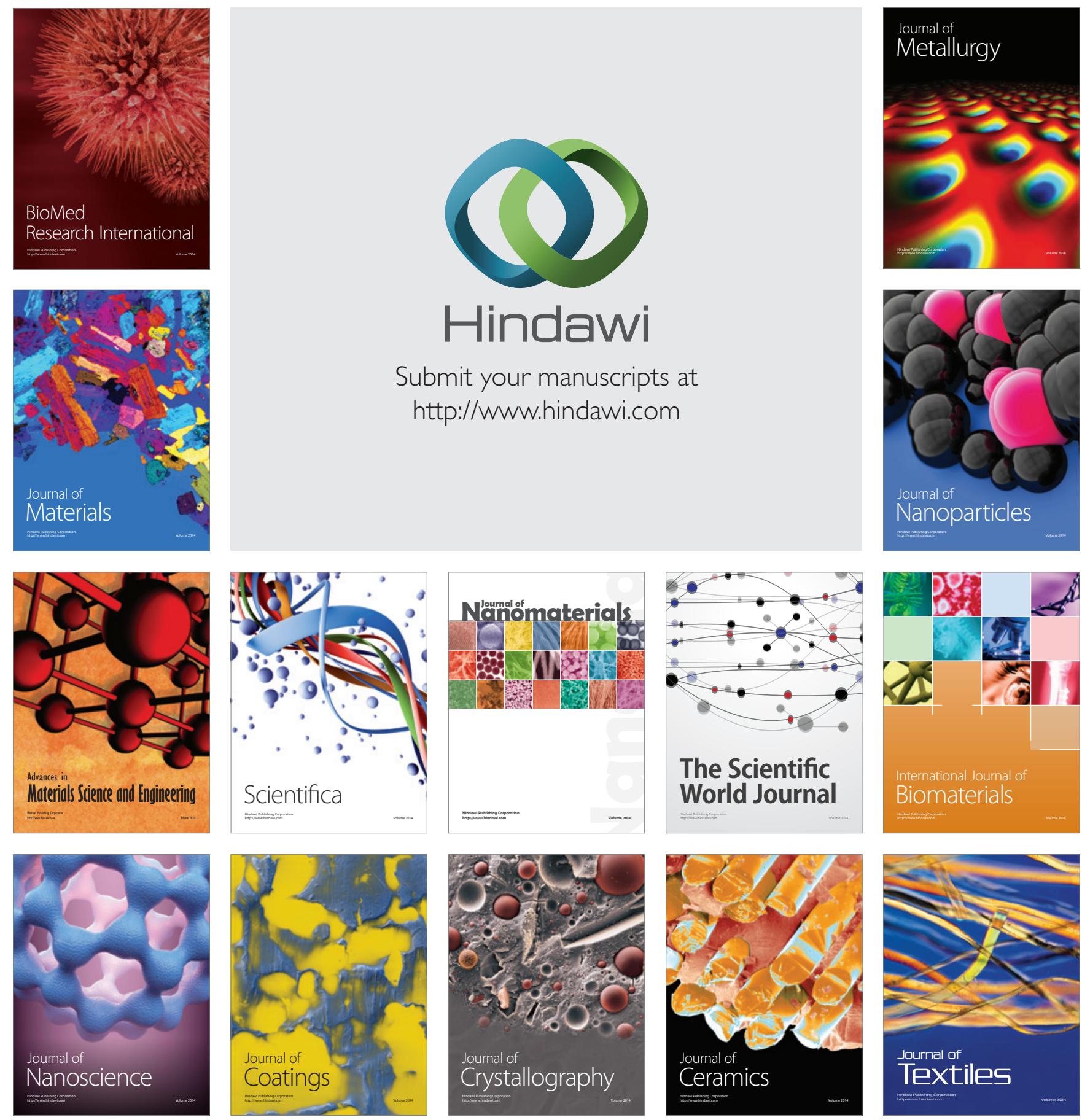